\title{
Main Problems and Tasks of Intellectualisation of Information Processing System
}

\author{
Mukhamedieva D.T., Safarova L.U.
}

\begin{abstract}
Semistructured decision-making systems (SDMS) are an important class of intelligent systems (IS) in conditions of different types of indeterminacy and, fuzzy indeterminacy in particular. A dominating issue in projection of indistinct logical system is a selection of intelligent database, or rather a rational number of rules and effective values of their membership function. As a result of it the main problems and tasks of information processing system intellectualisation and their ways of solution are presented in this article.
\end{abstract}

Keywords: Information processing, Semistructured decisionmaking systems, intelligent systems, fuzzy sets

\section{INTRODUCTION}

Obtained results in the field of artificial brain $(\mathrm{AB})$ allow putting and solving issues associated with the construction of complex structured information processing of IS and its recognition [1-4]. One of the major theoretical and applicable problems of IS construction is the development of formal technique of intelligent behaviour theoretical model making as well as IS application means of construction, where theoretical models are fulfilled, and their adequacy is audited [5-7].

When building mathematical models researchers are often faced with the problem of complex process model development defined by diverse types of indeterminacy. In such situations they have to select from two options: either to consider the influence of indeterminacy as insignificant that have been led to the loss of information and reduction of model adequacy to the real process or complicate the mathematical models. In practice, they have to deal with data that is not agreed with assumptions underlying the classical approaches. Nowadays no one doubts that there is the necessity to assess indeterminacies at the time of modelling and regulating the real processes. At the same time the restriction awareness of classical probabilitytheoretical approach to the indeterminacy interpretation has brought to a great number of alternative theories and methods. The theory of fuzzy sets can be emphasized among them [1-7].

Revised Manuscript Received on July 25, 2019

Mukhamedieva D.T, Tashkent university of information technologies Safarova L.U Samarkand agricultural institute, Samarqand, Uzbekistan.
In addition, new approaches do not deny the classical probability-theoretical methodology but rather supplement and expand it with the help of reasonable synthesis method that allows solving practical problems more effectively [812].

There are various types of indeterminacy:

1) Conceptual indeterminacy, for instance, in familiar situations of quantum mechanics;

2) Indeterminacy generated by a general number of objects and elements included in situation, for example, at the number of order elements of more than $10^{9}$;

3 ) indeterminacy caused by the deficiency and validity of information due to technical, social and other reasons; 4) indeterminacy, arising from unreasonable pay for distinctness achievement;

5) Indeterminacy, arising from decision-maker due to deficiency of experience and knowledge of factors having an effect on decision-making;

6) Indeterminacy connected with restrictions in situation of decision-making (time limits and parametric space identifying factors of decision-making);

7) Indeterminacy caused by environmental behaviour or opponent that has an influence on the decision-making process $[13,14]$.

\section{MAIN PROBLEMS OF INTELLECTUALISATION OF INFORMATION PROCESSING SYSTEM}

There is an array of situations in the decision-making processes possessing one or another level of indeterminacy and requiring for getting decision for its description of such mathematical tool which would include an opportunity of indeterminacy appearance.

Historically the first tool of its kind was an apparatus of formal logic and probability calculus in compliance with which the uncertainty of the situation is described as normalized measure defining an opportunity of specified chances outcome given in advance (elements and subsets of some sets). The development of probabilistic methods description of uncertain situations is the game theory of statistic decision. Basically, these theories can be considered as the extreme cases of various levels of indeterminacy gradation in informational issues.

Formal logic is used for exception inaccuracy and ambiguity from reasoning. However it operates only with clear concepts. Therefore actuality has become a necessity of theoretical and applicable apparatus creation which helps to describe formally nonstrict, fuzzy concepts and supplying an opportunity to make procedure realisation of logical deduction and reasoning more effective that contain indistinct ideas. A 
substantial contribution in this direction has become an approach based on the use of concept of indistinct, degraded, fuzzy sets introduced by L.Zadeh [1-4]. This instrument is an adequate for description of such kind of situations which do not have clearly defined boundaries. Ziman [5-7] who presented tolerance relation expressing intuitive notion of similarities (indistinguishability) in pure mathematical form, used another approach to describe indistinct cases. Tolerance relation characterises categories of objects with degraded, fuzzy boundaries as well. Close to Zadeh's point of view is Vatanabe's approach [7] who builds up foundations of logic on the base of generic function defining a certain class of indeterminate cases.

The development of the fuzzy sets theory and its use in different disciplines are connected with the names of such famous scientists as R. Bellman, A.Kofman, as well as L.A. Zadeh [1-5]. The theory of fuzzy sets (TFS) and fuzzy logic experienced their second birth in the early eighties of $\mathrm{XX}$ century when some groups of researchers (especially in the USA and Japan) focused on the development of computer systems of different applications using fuzzy managing algorithms. Theoretical foundations for these development had been laid in D. Dubois and A. Prada's works in the theory of possibilities [4], R. Jager's in the theory of fuzzy operators [2], M. Sugeno's in the theory of fuzzy measure and fuzzy integrals, E. Mamdani and E. Sanches's piece of work in the theory of fuzzy relations, T. Saati and other mathematicians' works [6,7]. A. Kofman's monograph [5] is emphasized particularly as a systematization of outcomes in all spheres of fuzzy mathematics.

Some conceptual works are highlighted among last investigations: L.Zadeh - on transition from numerical computing to lexical computing and the role of natural language in data processing, decision-making and managing; M. Jamshidi - on soft computing application, neural networks and evolutional calculations in the industrial systems; B. Fazlollahi - on decision-making support systems in marketing investigations, R. Alieva - on intellectual hybrid systems [1-7]; $\quad$ S. Ulyanova - robust intelligent control systems in unpredictable situations based on indistinct quantum situations and self-organization algorithms of knowledge bases [1-4]. The given statements, for example, № [1] about fuzzy and multiple approach within the next few years will be one of the main mathematical tools in various fields of human knowledge which is defined as follows:

- in the philosophical sense this approach disclose new conceptual opportunities for problem solving of abstraction and concept of formation which have a richness of all kinds of shades;

- the field of large system analysis discovers an opportunity of indeterminacy modelling expressed especially in the awareness gradation of top echelon in hierarchical systems on downgrading levels;

- an opportunity in the modelling of integrity features, diffuseness of mental imagery, mental flexibility, multivaluedness of language elements existed at all reflection levels, regulation and communication appears in the field of psychology;

- an opportunity of the meaning of sentences and texts modelling is given in the field of linguistics which is made by means of opportunities distribution described by membership functions;
- a possibility of modelling the elements of teaching and educational process, use of qualitative characteristics of various pedagogical phenomena, still "not measured" measurements of personality traits, such as good breeding, learning ability, perspective, talent are opened in the field of pedagogics.

Fuzzy and multiple approach allows receiving the strict mathematical description of indistinct statements overcoming a linguistic barrier between the person to some extent, judgements where assessments are approximate and indistinct, and COMPUTERS which can produce only accurate instructions. A man is able to argue, learn and take decisions in fuzzy, indistinct condition. Possibilities of modern COMPUTERS are far from opportunities of a person. Therefore, nowadays indistinct and multiple approach is widely used at representation and parameters assessment, and a component of any complex system as "fuzziness" by the opportunities is adequate to abilities and human intelligence.

At the same time, despite the achieved success, many means of mathematical tool of the fuzzy sets theory (FST) and fuzzy inference (FI) require further development as long as by now they have not reached the level of the traditional mathematics apparatus yet. Development of fuzzy and multiple approach will allow improving the theoretical and applied tool for the development of more effective methods of the decision of hardly formalizable tasks with indistinct parameters. It will allow expanding application of this approach in various spheres: scientific, technical, social, etc.

At the solution of issues in the deterministic formulation with growth of model complexity and dimension there are big problems with stability of optimization problems. Process of optimization means a system conclusion to certain limit restrictions. In this situation, even insignificant fluctuations of minor parameters can lead to the loss of the mode. It is impossible to expand simply these restrictions; the procedure of optimization right there will lead the mode to new restrictions and the problem of stability will remain. Therefore, only representation of a number of restrictions as fuzzy gives the chance to receive a steady decision in the conditions of information inaccuracy and fuzziness of production restrictions with an indication of admissibility decrease of this mode.

The main issues of problem solution of nonlinear optimization at adoption of semistructured decisions in the fuzziness conditions of initial information (parameters and coefficients of criteria and restrictions) are: finding of analytical solutions; development of computing algorithms; application of interval and fuzzy arithmetic allowing operation during problem solving of optimization with the areas of admissible decisions (with a changing degree of admissibility) [8-14].

The results of researches stated in the subsequent sections are directed to the solution of some of the specified problems of intellectual systems creation in the processing of complicated 
structure of fuzzy information in the adoption of semistructured decisions systems (ASDS) [15-18].

\section{CREATION OF APPLIED INTELLIGENT PROCESSING SYSTEMS OF COMPLICATED STRUCTURE OF FUZZY INFORMATION}

Potentially-enable yield with the adjustment under normal weather conditions and available water supply is determined by formula $\bar{y}=y(1+w)$, where $\mathrm{w}$ - restoring coefficient of short received yield due to unfavourable weather conditions and water availability.

Forecasting yield for the next year taking into account water supply and weather conditions of the current year is defined by formula $y^{\Pi}=\bar{y}\left(1-v_{i}\right)$, where $v_{i}$ - forecasting coefficient.

Let's take a detailed look at the yield of mineral and organic fertilizers.

Mineral and organic fertilizers are the most significant factors guaranteeing continuous high-yielding crop. Moreover, they support and increase soil fertility. Each kilogram of mineral fertilizer brought to crops provides a bigger harvest. It can be assumed that expansion in the number of brought mineral fertilizer by $\Delta M$ will provide a yield increase at $\Delta y$.

A forecasting yield for the year of projection taking into account water supply, weather conditions and a difference of mineral fertilizers is defined by $\mathrm{c} / \mathrm{ha}$ :

$$
y^{T}=y^{\Pi}+\Delta y \text {. }
$$

When modelling the yield forecast of a cotton plant in the fuzzy environment we use the following designations:

$P_{k i j}$ - cultivated area of a cotton plant, ha;

$Y_{k i j}$ - cotton yield, c/ha;

$\mu Y_{k i j}$ - membership function for a cotton yield;

$C_{k i j}$ - selection variety;
$N_{k i j}$ - application of nitrogen amount for a cotton plant, $\mathrm{kg} / \mathrm{ha}$;

$$
\mu N_{k i j} \text { - membership function for applied amount }
$$
of nitrogen under a cotton plant;

$B O_{k i}$ - available water supply;

$\mu B O_{k i}$ - membership function for water supply;

$\Pi_{k i}$ - weather conditions of the sowing period;

$\mu \Pi_{k i^{-}}$membership function for weather conditions of the sowing period;

$B_{k i}$ - weather conditions of the vegetative period;

$\mu B_{k i}$ - membership function for weather conditions of the vegetative period;

$Y b_{k i}$ - weather conditions of the harvest period;

$\mu Y S_{k i}{ }^{-}$membership function of weather conditions of the harvest period;

$\mathrm{k}$-the years preceding forecasting;

$i=\overline{1, n}-$ a number of the object;

$\mathrm{j}$ - an index of a selection variety of a cotton plant.

The forecast of the cotton plant yield is carried out by the restoration method of potentially possible harvest halfreceived because of the influence of unfavourable weather conditions and water supply during sowing, vegetation and harvesting, by introduction of restoring coefficient. It is appropriate to use fuzzy mathematics methods as weather conditions, yield, water supply are fuzzy numbers [1].

Potentially possible yield with improvement under normal weather conditions and water supply is determined by the next formula:

$$
\bar{Y}_{k i j}=\left(\sum_{s=1}^{m} \mu^{s} Y_{k i j} Y_{k i j}^{s} / \sum_{r=1}^{m} \mu^{r} Y_{k i j}\right)\left(1+w_{k i}\right),
$$

where $w_{k i^{-}}$restoring coefficient of the half-received harvest because of unfavourable weather conditions and water supply. 
Restoring coefficient is expressed by the next formula:

$$
\begin{gathered}
w_{k i}=0,01 \rho_{1}\left(1-\sum_{s=1}^{m} \mu \Pi_{k i}^{s} \Pi_{k i}^{s} / \sum_{r=1}^{m} \mu \Pi_{k i}^{r}\right) * \\
*\left(1-0,3 \sum_{s=1}^{m} \mu B O_{k i}^{s} B O_{k i}^{s} / \sum_{r=1}^{m} \mu B O_{k i}^{r}-0,7 \sum_{s=1}^{m} \mu B_{k i}^{s} B_{k i}^{s} / \sum_{r=1}^{m} \mu B_{k i}^{r}\right)+ \\
+0,01 \rho_{2}\left(1-\sum \mu B_{k i}^{s} B_{k i}^{s} / \sum_{r=1}^{m} \mu B_{k i}^{r}\right)+0,01 \rho_{4}\left(1-\sum_{s=1}^{m} \mu B O_{k i}^{s} B O_{k i}^{s} / \sum_{r=1}^{m} \mu B O_{k i}^{r}\right) * \\
*\left(1-0,4 \sum_{s=1}^{m} \mu B_{k i}^{s} B_{k i}^{s} / \sum_{r=1}^{m} \mu B_{k i}^{r}-0,2 \sum_{s=1}^{m} \mu \Pi_{k i}^{s} \Pi_{k i}^{s} / \sum_{r=1}^{m} \mu \Pi_{k i}^{r}\right)+ \\
+0,01 \rho_{3}\left(1-\sum_{s=1}^{m} \mu Y B_{k i}^{s} / \sum_{r=1}^{m} \mu Y B_{k i}^{r}\right) .
\end{gathered}
$$

Here the effect of impact factor on reduction in yields is caused by weather conditions at sowing at $\rho_{1} \%$, at vegetation $-\rho_{2} \%$, at harvesting- $\rho_{3} \%$ and at water insecurity $-\rho_{4} \%$.

The impact of weather conditions is defined on the basis of long-term observations and takes the next value

$$
\begin{gathered}
\rho_{i}=\sum_{s=1}^{m} \rho_{i}^{s} \mu \rho_{i}^{s} / \sum_{r=1}^{m} \mu \rho_{i}^{r}, \quad i=\overline{1,4} \\
\mu \rho_{1}^{s}=1 /(1+|\rho-4|) ; \\
\mu \rho_{3}^{s}=1 /(1+|\rho-10|) ; \\
\mu \rho_{2}^{s}=1 /(1+|\rho-7|) ;
\end{gathered}
$$$$
\mu \rho_{4}^{s}=1 /(1+|\rho-12|) \text {. }
$$

If weather conditions during sowing are favourable then the decrease in the cotton yield is not expected. On the contrary, under unfavourable weather conditions when sowing the productivity of a cotton plant

decreases by $\rho_{1} \%$ depending on future weather conditions at the vegetation period and water supply. Let us suppose that possible fall in the cotton yield $\rho_{1} \%$ can be compensated by normal water supply, favourable weather conditions in the vegetation period with implementation of a timely set of agrotechnical actions due to bad weather conditions in the sowing period. If water supply is well below normal (at the water deficiency), and weather conditions in the vegetation period are favourable (i.e. conditions of carrying-out agrotechnical measures are normal), then influence of bad weather conditions during sowing will reach $0,003 \rho_{1} \%$. The effect of bad weather conditions on the cotton yield at the normal water supply in the vegetation period during sowing will make $0,007 \rho_{1}$. The effect of bad weather conditions at the water deficiency during sowing reduces productivity on $0,01 \rho_{1}$ in the vegetation period.

If weather conditions in the vegetation period are favourable then they do not influence on the decrease of

the cotton yield. The effect of bad weather conditions in the vegetation period on the yield will compose $0,01 \rho_{2}$.

If water supply is considered normal then influence on the yield does not happen. At water supply deficiency, the influence on the cotton yield decrease can be reduced due to the rational use of water resources. Under favourable weather conditions when sowing in the vegetation period the influence of water supply on the yield will make $0,004 \rho_{4}$ where it is supposed to reduce the negative effect on the yield due to the effective watering and high-quality performance of agrotechnical actions.

If weather conditions in the vegetation period are favourable but conditions when sowing are adverse, the influence of water supply on productivity will compose $0,006 \rho_{4}$. On the contrary, if weather conditions are adverse in the vegetation period and conditions when sowing are favourable, then influence of water security on productivity makes $0,008 \rho_{4}$. Under adverse weather conditions during sowing and in the vegetation period influence of water supply on productivity will make 0,01 $\rho_{4}$. The loss productivity is not expected under favourable weather conditions during the harvest period. If conditions are adverse in the harvest period then their influence on productivity will make $0,01 \rho_{3}$.

Thus, we have described various options of influence on decrease in cotton productivity, water supply factors and weather conditions during the sowing, vegetation and in the harvest period.

The forecasting yield including water supply and weather conditions of the current year in the indistinct environment expresses:

$$
Y_{k i j}^{\Pi}=\left(\sum_{s=1}^{m} \bar{Y}_{k i j}^{s} \mu Y_{k i j}^{S} / \sum_{r=1}^{m} \mu Y_{k i j}^{r}\right)\left(1-v_{i}\right) \text {, }
$$

where $v_{i}$ - forecasting coefficient is defined by the formula: 


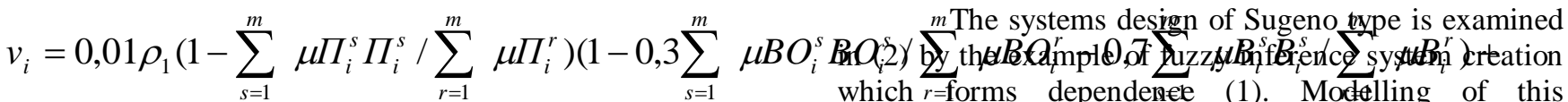
dependence is carried out by means of the knowledge $+0,01 \rho_{2}\left(1-\sum_{s=1}^{m} \mu B_{i}^{s} B_{i}^{s} / \sum_{r=1}^{m} \mu B_{i}^{r}\right)+$ base shown in the tab. 1.

$+0,01 \rho_{4}\left(1-\sum_{s=1}^{m} \mu B O_{i}^{s} B O_{i}^{s} / \sum_{r=1}^{m} \mu B O_{i}^{r}\right)\left(1-0,4 \sum_{s=1}^{m}\right.$ $+0,01 \rho_{3}\left(1-\sum_{s=1}^{m} \mu Y S_{i}^{s} Y S_{i}^{s} / \sum_{r=1}^{m} \mu Y B_{i}^{r}\right)$.

The effect of fertilizers on productivity depends on water supply and weather conditions in the vegetation period. At normal water supply and favourable weather conditions nutrients acclimate in the vegetation period at $\delta \%$, where $\delta=40 \%$ from the whole amount of applied nitrogen. In addition, the effect of nutrients on creation of cotton yield decreases at water deficiency and deterioration of weather conditions. Let reduction of nutrients effects make $\gamma \%$. The $\gamma$-reduction occurs at $\alpha \%$ due to water deficiency and $\beta \%$ due to adversity of weather conditions in the vegetation period.

We take into consideration an object (1) for which connection «entrances $x_{i}$ - exit y» can be presented in the form of the knowledge-based matrix shown in the table F5.2. The fuzzy knowledge base corresponds to this matrix:

IF

$\left[\left(x_{1}=a_{1}^{j 1}\right) И\left(x_{2}=a_{2}^{j 1}\right) И \ldots И\left(x_{n}=a_{n}^{j 1}\right)\right]$

(weighted $w_{j 1}$ )

OR

$\left[\left(x_{1}=a_{1}^{j 2}\right) И\left(x_{2}=a_{2}^{j 2}\right) И \ldots И\left(x_{n}=a_{n}^{j 2}\right)\right]$

(weighted $\left.w_{j 2}\right) \ldots$

$\left[\left(x_{1}=a_{1}^{j k_{j}}\right) И\left(x_{2}=a_{2}^{j k_{j}}\right) И \ldots И\left(x_{n}=a_{n}^{j k_{j}}\right)\right]$ (weighted $w_{j k_{j}}$ ),

THEN

$y_{j}=c_{o}^{j p}+c_{1}^{j p} x_{1}^{j p}+\ldots+c_{n}^{j p} x_{n}^{j p} \quad$ for each

$j=\overline{1, m}$

Where $a_{i}^{j p}$ - a linguistic term which evaluates a variable $x_{i}$ in line with number $p=\overline{1, k_{j}} ; k_{j-}$ number of conjunction lines corresponding to a class $d_{j}, j=1, m ; w_{j p}$ - number in the range of $[0,1]$ which describes expression weight with number jp [1-6].

It is possible to assume that increase in the amount of applied fertilizer for $\triangle N P K$ supplies additional

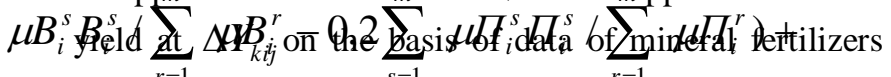
consumption for cotton harvesting. Taking into account water supply and weather conditions in the vegetation period where the correction factor of productivity on mineral fertilizers will make:

$$
\begin{aligned}
& \Delta Y_{k i j}=\lambda \cdot \delta\left\{N P K_{i}\left[1-0,0001 \gamma\left(\alpha\left(B O_{i}-1\right)+\beta\left(B_{i}-1\right)\right)\right]-\right. \\
& \left.-N P K_{k i}\left[1-0,0001 \gamma\left(\alpha\left(B O_{k i}-1\right)+\beta\left(B_{k i}-1\right)\right)\right]\right\}
\end{aligned}
$$

The $\alpha, \beta, \delta, \gamma, \lambda$ - fuzzy numbers:

$$
\begin{gathered}
\lambda=\sum_{s=1}^{m} \mu_{l}^{s} \lambda_{l}^{s} / \sum_{r=1}^{m} \mu_{l}^{r}, \\
\delta=\sum_{s=1}^{m} \mu_{d}^{s} \delta_{d}^{s} / \sum_{r=1}^{m} \mu_{d}^{r}, \\
\alpha=\sum_{s=1}^{m} \mu_{a}^{s} \alpha_{a}^{s} / \sum_{r=1}^{m} \mu_{a}^{r}, \\
\beta=\sum_{s=1}^{m} \mu_{b}^{s} b_{b}^{s} / \sum_{r=1}^{m} \mu_{b}^{r}, \\
\gamma=\sum_{s=1}^{m} \mu_{g}^{s} \gamma_{g}^{s} / \sum_{r=1}^{m} \mu_{g}^{r}
\end{gathered}
$$

Here

$$
\begin{gathered}
\mu_{a}=1 /(1+|\alpha-60|), \\
\mu_{b}=1 /(1+|\beta-25|), \\
\mu_{g}=1 /(1+|\gamma-30|), \\
\mu_{d}=1 /(1+|\delta-0,4|) .
\end{gathered}
$$

$\lambda$ depends on the type of soil, fertilizer rate, growth of cotton plant.

On watered typical sierozem soil at application of fertilizer rate N-200, $\mathrm{P}_{2} \mathrm{O}_{5}-140, \mathrm{~K}_{2} \mathrm{O}-100 \mathrm{~kg} / \mathrm{ha}$ the growth C-4727 will provide an additional yield $\lambda \mathrm{c} / \mathrm{ha}$,

$$
\mu_{l}=1 /(1+|\lambda-21,7|)
$$

the Tashkent growth -1 will provide yield increase $\lambda \mathrm{c} / \mathrm{ha}$,

Published By: 


$$
\mu_{l}=1 /(1+|\lambda-15,6|),
$$

the growth $108-\mathrm{F}$ will provide yield increase $\lambda$ c/ha,

$$
\mu_{l}=1 /(1+|\lambda-17,7|),
$$
$\lambda \mathrm{c} / \mathrm{ha}$,

the growth 159-F will provide an additional yield

$$
\mu_{l}=1 /(1+|\lambda-11,8|) .
$$

On watered typical sierozem and meadowy soil at application of fertilizer rate N-250, $\mathrm{P}_{2} \mathrm{O}_{5}-175, \mathrm{~K}_{2} \mathrm{O}$ $125 \mathrm{~kg} / \mathrm{ha}$ the growth $\mathrm{C}-4727$ will provide an additional yield $\lambda \mathrm{c} / \mathrm{ha}$,

$$
\mu_{l}=1 /(1+|\lambda-25,1|),
$$

Table 1: Knowledge-based matrix of forecasting cotton yield

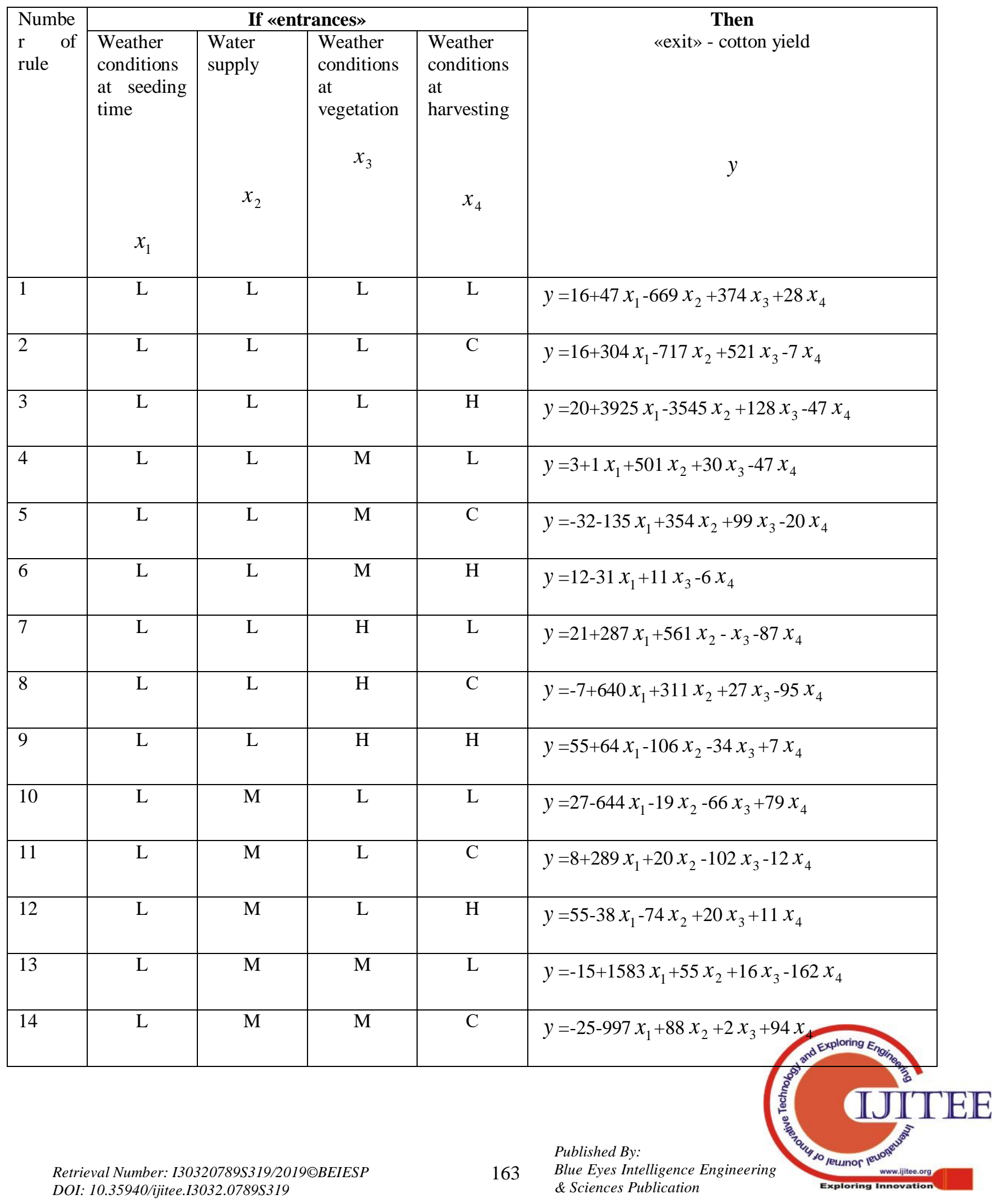




\begin{tabular}{|c|c|c|c|c|c|}
\hline 15 & $\mathrm{~L}$ & $\mathrm{M}$ & $\mathrm{M}$ & $\mathrm{H}$ & $y=-63-7 x_{1}-12 x_{2}+179 x_{3}-16 x_{4}$ \\
\hline 16 & $\mathrm{~L}$ & $\mathrm{M}$ & $\mathrm{H}$ & $\mathrm{L}$ & $y=16+1240 x_{1}-3 x_{2}+7 x_{3}-120 x_{4}$ \\
\hline 17 & $\mathrm{~L}$ & $\mathrm{M}$ & $\mathrm{H}$ & $\mathrm{C}$ & $y=35+317 x_{1}-19 x_{2}-4 x_{3}-28 x_{4}$ \\
\hline 18 & $\mathrm{~L}$ & $\mathrm{M}$ & $\mathrm{H}$ & $\mathrm{H}$ & $y=85-223 x_{1}-55 x_{2}-36 x_{3}+26 x_{4}$ \\
\hline 19 & $\mathrm{~L}$ & $\mathrm{H}$ & $\mathrm{L}$ & $\mathrm{L}$ & $y=-5+655 x_{1}+25 x_{2}-700 x_{3}-53 x_{4}$ \\
\hline 20 & $\mathrm{~L}$ & $\mathrm{H}$ & $\mathrm{L}$ & $\mathrm{C}$ & $y=-104 x_{1}+20 x_{2}-2278 x_{3}+37 x_{4}$ \\
\hline 21 & $\mathrm{~L}$ & $\mathrm{H}$ & $\mathrm{L}$ & $\mathrm{H}$ & $y=113 x_{1}+20 x_{2}+1779 x_{3}-25 x_{4}$ \\
\hline 22 & $\mathrm{~L}$ & $\mathrm{H}$ & $\mathrm{M}$ & $\mathrm{L}$ & $y=-13-1630 x_{1}+11 x_{2}+47 x_{3}+161 x_{4}$ \\
\hline 23 & $\mathrm{~L}$ & $\mathrm{H}$ & $\mathrm{M}$ & $\mathrm{C}$ & $y=48+1099 x_{1}+16 x_{2}-86 x_{3}-97 x_{4}$ \\
\hline 24 & $\mathrm{~L}$ & $\mathrm{H}$ & $\mathrm{M}$ & $\mathrm{H}$ & $y=-10+84 x_{1}+21 x_{2}+20 x_{3}-6 x_{4}$ \\
\hline 25 & $\mathrm{~L}$ & $\mathrm{H}$ & $\mathrm{H}$ & $\mathrm{L}$ & $y=-386 x_{1}+20 x_{2}+2 x_{3}+43 x_{4}$ \\
\hline 26 & $\mathrm{~L}$ & $\mathrm{H}$ & $\mathrm{H}$ & $\mathrm{C}$ & $y=-27+139 x_{1}+22 x_{2}+27 x_{3}-7 x_{4}$ \\
\hline 27 & $\mathrm{~L}$ & $\mathrm{H}$ & $\mathrm{H}$ & $\mathrm{H}$ & $\begin{aligned} y= & 7499-30600 x_{1}-4378 x_{2}-3071 x_{3} \\
& +2317 x_{4}\end{aligned}$ \\
\hline
\end{tabular}

\section{CONCLUSION}

A dominating issue in projection of fuzzy logical system is a selection of intelligent database, or rather a rational number of rules and effective values of their membership function. Various ways of formation of fuzzy knowledge bases are known as: acquiring of knowledge from the expert, the way based on the use of mathematical models, neuro-fuzzy approach, etc. At the same time as the main criteria always act the absence of redundancy of fuzzy rules, discrepancy as well as defective, false knowledge. The designers of indistinct logical systems usually try to receive the acceptable solution at the minimum quantity of fuzzy rules. It is especially important from the point of view of effective implementation of fuzzy hardware and fuzzy software of the designing system. One of the solutions of this problem, i.e. the effective network design of fuzzy rules is the approach based on the consumption of neural networks and evolutionary algorithms.

Realization of system provides:

- improvement of adoption quality of group decisions in the conditions of various situations emergence due to computer decision-making and machine experiment with imitation of the respective situation;

- possibility of the development of administrative decisions and recommendations allowing reduction of human and material losses;

- economy of resources (material, labour) due to modelling of the collective decisions adoption on the computer, diversity of the worked out decisions and effective use in real conditions of pre-arranged decisions; - increase in the effectiveness of training on the basis of use of the modern computer aids and software, mathematical methods and software applications.

The used mathematical tool is rather labour intensive regarding computing procedures. Therefore, the efficiency of its application is reached in the presence of special computer developments.

\section{REFERENCES}

1. Zadeh L.A. Fuzzy sets: Inform. and Control. 1965, №8, pp. 338353.

2. Yager R.R., Zadeh L.A. (Eds.), Fuzzy sets, neural networks and Soft 
Computing, VAN Nostrand Reinhold, New York, 1994, 440p.

3. Aliev R.A., Barak D, Chew G.at all, SOFT COMPUTING: Fuzzy Logic. Neural Networks and Distributed Artificial Intelligence. F.Amin zadeh. Jamshidi M. (Eds.), PTR Prentice Hall Englewood Cliffs, New Jer sey, 1994, 301p.

4. Dubois D., Prade H. Fuzzy sets and systems: Theory and applications. New York: Acad. Press, 1980,394p.

5. Kaufmann A., Gupta M.M. Introduction to fuzzy Arifmetic, New York: Van Hostrand, 1985.

6. Sugeno M. Fuzzy measures and fuzzy integrals.- A survey. In Gupta, Saridis, Gaines, 1977, pp. 89-102.

7. Mamdani E.H. Applications of fuzzy logic to approximate reasoning using linguistic systems: IEEE Transactions on Computer C-26, 1977. pp. 1182-1191.

8. Hopfield J.J. Neural Network and Physical Systems Akademy of Science 79, 1982, pp. 2554-2559.

9. Hopfield J.J. Neurons with Gaded Response Have Collective Computational Properties Like Those of Two-state Neurons. Proc. of the National Academy of Science 81, 1984, pp. 3088-3092.

10. Kosko B. Neural Networks and Fuzzy Systems. A dynamical System Approach to Machine Intelligence. Prentice-Hall International Inc., 1993, 449.

11. Hebb D.O.: The Organization of Behavior. Willey, New York, 1994.

12. Genetic Algorithms and Simulated Annealing, L. Davis (Eds), Pitman, London. 1987.

13. Holland S.H. Adaptation in Natural Artificial Systems, university of Michigan Press, Ann Arbor. MI, 1975.

14. Lee M., Lee S.Y., Park C.H. Neuro-fuzzy Identification model of nonlinear Dynamic Systems. Proc. of the 2 and Inter. Conf. on Fuzzy Logic and Neural Networks., vol.1, 1992, pp.485-488.

15. Muxamediyeva D.T. Model of estimation of success of geological exploration perspective // International Journal of Mechanical and production engineering research and development (IJMPERD) ISSN(P): 2249-6890; ISSN(E): 2249-8001 Vol. 8, Issue 2, USA. 2018, 527-538 pp. Impact Factor (JCC): 6.8765.

16. Muxamediyeva D.K. Properties of self similar solutions of reactiondiffusion systems of quasilinear equations // International Journal of Mechanical and production engineering research and development (IJMPERD) ISSN(P): 2249-6890; ISSN(E): 2249-8001 Vol. 8, Issue 2, USA. 2018, 555-565 pp. Impact Factor (JCC): 6.8765.

17. Muxamediyeva D.T. Structure of fuzzy control module with neural network //International Journal of Mechanical and Production Engineering Research and Development (IJMPERD) ISSN (P): 2249-6890; ISSN (E): 2249-8001 Vol. 9, Issue 2, Apr 2019, pp.649658

18. Muxamediyeva D.K. The property of the problem of reaction diffusion with double nonlinearity at the given initial conditions //International Journal of Mechanical and Production Engineering Research and Development (IJMPERD) ISSN (P): 2249-6890; ISSN (E): 2249-8001 Vol. 9, Issue 3, Jun 2019, pp.1095-1106 